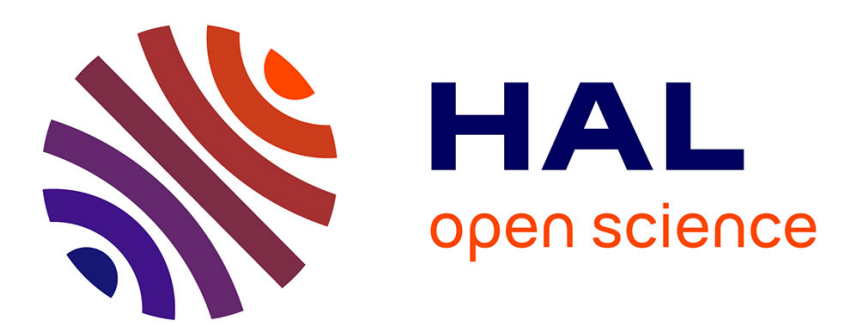

\title{
A general adaptive feedforward compensation algorithm for active vibration control with mechanical coupling and local feedback
}

Ioan Doré Landau, Tudor-Bogdan Airimitoaie

\section{- To cite this version:}

Ioan Doré Landau, Tudor-Bogdan Airimitoaie. A general adaptive feedforward compensation algorithm for active vibration control with mechanical coupling and local feedback. MED 2014 - 22nd Mediterranean Conference on Control and Automation, Mediteranean Control Association, Jun 2014, Palermo, Italy. pp.67-72, 10.1109/MED.2014.6961328 . hal-01394158

\section{HAL Id: hal-01394158 \\ https://hal.science/hal-01394158}

Submitted on 16 Nov 2016

HAL is a multi-disciplinary open access archive for the deposit and dissemination of scientific research documents, whether they are published or not. The documents may come from teaching and research institutions in France or abroad, or from public or private research centers.
L'archive ouverte pluridisciplinaire HAL, est destinée au dépôt et à la diffusion de documents scientifiques de niveau recherche, publiés ou non, émanant des établissements d'enseignement et de recherche français ou étrangers, des laboratoires publics ou privés. 


\title{
A General Adaptive Feedforward Compensation Algorithm for Active Vibration Control with Mechanical Coupling and Local Feedback
}

\author{
Ioan Doré Landau ${ }^{1}$ and Tudor-Bogdan Airimiţoaie ${ }^{2}$
}

\begin{abstract}
Adaptive feedforward broadband vibration (or noise) compensation is currently used when a correlated measurement with the disturbance is available. Most of the active vibration control systems feature an internal "positive" mechanical feedback between the compensation system and the reference source (a correlated measurement with the disturbance). Such systems have often also a feedback control loop for reducing the effect of disturbances. For stability reasons the adaptation algorithms require the implementation of a filter on observed data or a filtering of the residual acceleration in order to satisfy some passivity conditions. Recently the use of the "Integral + Proportional" (IP) adaptation has been proposed. The paper provides a general algorithms for the adaptive feedforward compensation which includes as particular cases the various algorithms presented in the literature. The algorithm has been applied to an active vibration control system.
\end{abstract}

\section{LIST OF ACRONYMS}

$\begin{array}{ll}\text { ANC } & \text { Active noise control } \\ \text { AVC } & \text { Active vibration control } \\ \text { FULMS } & \text { Filtered-u least mean squares } \\ \text { IIR } & \text { Infinite impulse response } \\ \text { IP } & \text { "Integral + proportional" } \\ \text { IP-PAA } & \text { "Integral + proportional" PAA } \\ \text { PAA } & \text { Parameter adaptation algorithm } \\ \text { PRBS } & \text { Pseudo random binary sequence } \\ \text { SPR } & \text { Strictly positive real (transfer function) }\end{array}$

\section{INTRODUCTION}

Adaptive feedforward for broadband disturbance compensation is widely used when a well correlated with the disturbance signal (image of the disturbance) is available ([1], [2], [3]). However, in many systems, there is a positive mechanical coupling between the feedforward compensation system and the measurement of the image of the disturbance. Using adaptation algorithms which did not take into account this internal coupling often leads to the instability of the system. In the context of this inherent "positive" feedback, the adaptive feedforward compensator should minimize the effect of the disturbance while simultaneously assuring the stability of the internal positive feedback loop [4].

Combining adaptive feedforward compensation with feedback control has been considered as an issue to further improve the performance of the adaptive feedforward compensation alone [5], [6], [7]. Unfortunately, there is a strong

\footnotetext{
${ }^{1}$ Ioan Doré Landau is with Control System Department of GIPSALab, University of Grenoble, St. Martin d'Héres, 38402 France ioan-dore. landau@gipsa-lab.grenoble-inp. fr

${ }^{2}$ Tudor-Bogdan Airimitgoaie is with the Fluminance team, IRISA / Inria, Campus Universitaire de Beaulieu, Rennes, 35042 France tudor-bogdan.airimitoaie@inria.fr
}

interaction between the presence of this local feedback controller and the stability conditions for the adaptive feedforward compensation algorithms[8].This interaction is further enhanced when the internal positive coupling is present.

Another important issue in adaptive feedforward compensation is the design of filters either on the observed variables of the feedforward compensator or on the residual acceleration in order to satisfy positive realness conditions on some transfer functions. In [4], it was shown that for small adaptation gains (slow adaptation) the violation of the positive real conditions in some frequency regions is acceptable, provided that in the average, the input-output product associated with this transfer function is positive, but the performance of the system is degraded. However, the problem of removing or relaxing the positive real condition can be also approached by adding a proportional adaptation to the widely used integral adaptation (see [9]). Another effect of the "Integral + Proportional" (IP) adaptation is that of speeding up the transients of the adaptation error.

A subject of debate in the context of adaptive feedforward compensation was the choice between filtering the data or filtering the residual acceleration (error) in order to satisfy the positive realness conditions required by the stability analysis (in the presence of the internal positive feedback or not). Some of the references discussing this issue are [10], [11], [12], [13]. As it will be shown, the reason to use one or both of the two options is related to the criterion which is minimized and to the presence or not of unstable zeros in the secondary path. The paper provides a general form for the adaptive feedforward compensation algorithms which includes as particular cases (almost) all adaptive feedforward IIR compensation algorithms available in the literature including recent developments. A stability analysis and experimental verifications are also included.

\section{An Active Vibration Control System Using AN INERTIAL ACTUATOR}

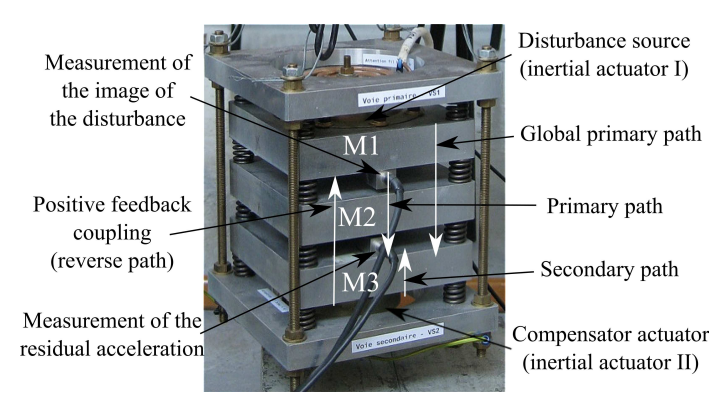

Fig. 1. The AVC system used for experimentations - photo. 


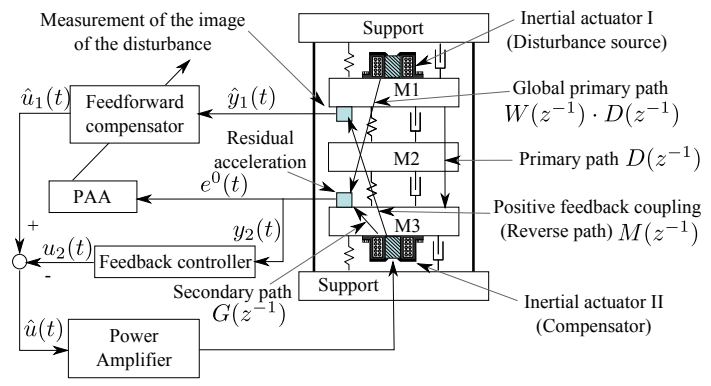

Fig. 2. An AVC system using an adaptive feedforward and a fixed feedback compensation scheme.

Figures 1 and 2 represent an AVC system using a measurement correlated with the disturbance and an inertial actuator for reducing the residual acceleration. The structure is representative for a number of situations encountered in practice. Details can be found in [4]

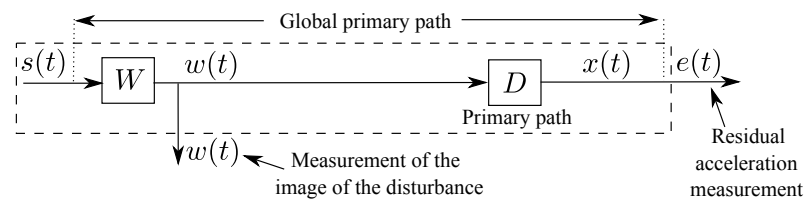

(a)

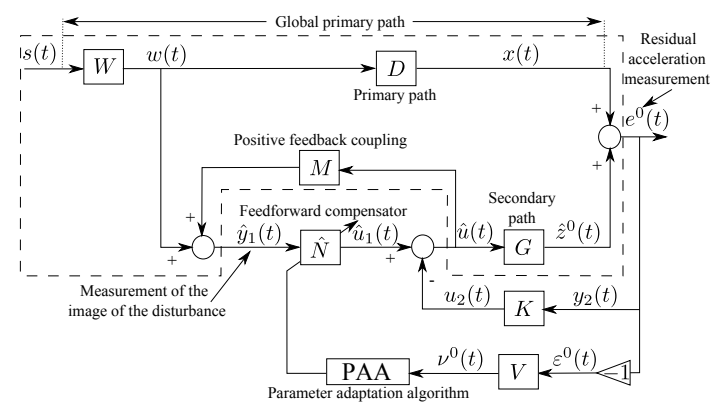

(b)

Fig. 3. Feedforward AVC: in open loop (a), with RS controller and adaptive feedforward compensator (b).

The corresponding block diagrams in open loop operation and with the hybrid (feedback-feedforward) compensation system are shown in Figures 3(a) and 3(b), respectively. In Figure $3(\mathrm{~b}), \hat{y}_{1}(t)$ denotes the effective output provided by the measurement device and which will serve as input to the adaptive feedforward filter $\hat{N}$. The control signal $\hat{u}(t)$, resulting from the difference between the output of the feedforward filter denoted by $\hat{u}_{1}(t)$ and the output of the feedback controller, is applied to the actuator through an amplifier. The transfer function $G$ (the secondary path) characterizes the dynamics from the control signal to the residual acceleration measurement $\left(e^{0}(t)\right)$ (amplifier + actuator + dynamics of the mechanical system). The transfer function $D$ between $w(t)$ and the measurement of the residual acceleration (in open loop operation) characterizes the primary path. The coupling between the control signal $\hat{u}(t)$ and the measurement $\hat{y}_{1}(t)$ through the compensator actuator is denoted by $M$. As indicated in Figure 3(b), this coupling is a "positive" feedback. This unwanted coupling raises problems in practice (source of instabilities) and makes the analysis of adaptive (estimation) algorithms more difficult. Reliable models of the various paths can be obtained by system identification techniques.

\section{BASIC EQUATIONS AND NOTATIONS}

The different blocks of the AVC system (Figure 3(b) are described in this section. The primary $(D)$, secondary $(G)$ and reverse (positive coupling) $(M)$ paths represented in 3(b) are respectively characterized by the asymptotically stable transfer operators:

$$
\begin{gathered}
D\left(q^{-1}\right)=\frac{B_{D}\left(q^{-1}\right)}{A_{D}\left(q^{-1}\right)}=\frac{b_{1}^{D} q^{-1}+\ldots+b_{n_{B_{D}}}^{D} q^{-n_{B_{D}}}}{1+a_{1}^{D} q^{-1}+\ldots+a_{n_{A_{D}}}^{D} q^{-n_{A_{D}}}}, \\
G\left(q^{-1}\right)=\frac{B_{G}\left(q^{-1}\right)}{A_{G}\left(q^{-1}\right)}=\frac{b_{1}^{G} q^{-1}+\ldots+b_{n_{B_{G}}^{G}}^{G} q^{-n_{B_{G}}}}{1+a_{1}^{G} q^{-1}+\ldots+a_{n_{A_{G}}^{G}}^{q^{-n_{A}}}}, \\
M\left(q^{-1}\right)=\frac{B_{M}\left(q^{-1}\right)}{A_{M}\left(q^{-1}\right)}=\frac{b_{1}^{M} q^{-1}+\ldots+b_{n_{B_{M}}^{M}}^{M} q^{-n_{B_{M}}}}{1+a_{1}^{M} q^{-1}+\ldots+a_{n_{A_{M}}^{M}}^{M} q^{-n_{A_{M}}}},
\end{gathered}
$$

with $B_{X}=q^{-1} B_{X}^{*}$ for any $x \in\{D, G, M\} . \hat{G}, \hat{M}$ and $\hat{D}$ denote the identified (estimated) models of G, M and D.

The optimal feedforward filter (unknown) is defined by

$$
N\left(q^{-1}\right)=\frac{R\left(q^{-1}\right)}{S\left(q^{-1}\right)}
$$

where

$$
\begin{gathered}
R\left(q^{-1}\right)=r_{0}+r_{1} q^{-1}+\ldots+r_{n_{R}} q^{-n_{R}}, \\
S\left(q^{-1}\right)=1+s_{1} q^{-1}+\ldots+s_{n_{S}} q^{-n_{S}}=1+q^{-1} S^{*}\left(q^{-1}\right) .
\end{gathered}
$$

The estimated feedforward filter is denoted by

$$
\hat{N}\left(q^{-1}\right)=\frac{\hat{R}\left(q^{-1}\right)}{\hat{S}\left(q^{-1}\right)} .
$$

The vector of optimal feedforward filter parameters is

$$
\Theta^{T}=\left[s_{1}, \ldots s_{n_{S}}, r_{0}, \ldots r_{n_{R}}\right]^{T}
$$

and the vector of estimated feedforward filter parameters is

$$
\hat{\boldsymbol{\Theta}}^{T}(t)=\left[\begin{array}{lllll}
\hat{s}_{1}(t), & \ldots & \hat{s}_{n_{S}}(t), \hat{r}_{0}(t), \ldots \hat{r}_{n_{R}}(t)
\end{array}\right]^{T} .
$$

The fixed feedback controller $K$, computed on the basis of the model $\hat{G}$ to reject broadband disturbances on the output $e^{0}(t)$, is characterized by the asymptotically stable transfer function

$$
K\left(q^{-1}\right)=\frac{B_{K}\left(q^{-1}\right)}{A_{K}\left(q^{-1}\right)},
$$

where

$$
\begin{aligned}
& B_{K}\left(q^{-1}\right)=b_{0}^{K}+b_{1}^{K} q^{-1}+\ldots+b_{n_{B_{K}}^{K}}^{K} q^{-n_{B_{K}}}, \\
& A_{K}\left(q^{-1}\right)=1+a_{1}^{K} q^{-1}+\ldots+a_{n_{A_{K}}^{K}}^{K} q^{-n_{A_{K}}} .
\end{aligned}
$$

The input of the feedforward filter (called also reference) is denoted by $\hat{y}_{1}(t)$ and it corresponds to the measurement provided by the primary transducer (force or acceleration transducer in AVC or a microphone in ANC). In the absence 
of the compensation loop (open loop operation), $\hat{y}_{1}(t)=$ $w(t)$. The output of the feedforward compensator is denoted by $\hat{u}_{1}(t+1)=\hat{u}_{1}(t+1 \mid \hat{\boldsymbol{\Theta}}(t+1))$ (a posteriori output) 1 . The measured input to the feedforward filter can also be written as

$$
\hat{y}_{1}(t+1)=w(t+1)+\frac{B_{M}^{*}\left(q^{-1}\right)}{A_{M}\left(q^{-1}\right)} \hat{u}(t),
$$

where

$$
\hat{u}=\hat{u}_{1}(t)-u_{2}(t),
$$

$\hat{u}_{1}(t)$ and $u_{2}(t)$ are the outputs given by the adaptive feedforward and the fixed feedback compensator, respectively. $\hat{u}$ is the effective input sent to the control actuator. The a priori output of the estimated feedforward filter is given by

$$
\begin{aligned}
\hat{u}_{1}^{0}(t+1) & =\hat{u}_{1}(t+1 \mid \hat{\boldsymbol{\Theta}}(t)) \\
& =-\hat{S}^{*}\left(t, q^{-1}\right) \hat{u}_{1}(t)+\hat{R}\left(t, q^{-1}\right) \hat{y}_{1}(t+1) \\
& =\hat{\boldsymbol{\Theta}}^{T}(t) \boldsymbol{\Phi}(t)=\left[\hat{\boldsymbol{\Theta}}_{S}^{T}(t), \hat{\boldsymbol{\Theta}}_{R}^{T}(t)\right]\left[\begin{array}{c}
\boldsymbol{\Phi}_{\hat{y}_{1}}(t) \\
\boldsymbol{\Phi}_{\hat{u}_{1}}(t)
\end{array}\right]
\end{aligned}
$$

where $\hat{\boldsymbol{\Theta}}^{T}(t)$ has been given in (9) and

$$
\begin{aligned}
\boldsymbol{\Phi}^{T}(t)= & {\left[\begin{array}{lll}
-\hat{u}_{1}(t), & \ldots-\hat{u}_{1}\left(t-n_{S}+1\right), \\
& \hat{y}_{1}(t+1), \hat{y}_{1}(t), \ldots \hat{y}_{1}\left(t-n_{R}+1\right)
\end{array}\right] } \\
= & {\left[\begin{array}{lll}
\boldsymbol{\Phi}_{\hat{u}_{1}}^{T}(t), & \boldsymbol{\Phi}_{\hat{y}_{1}}^{T}(t)
\end{array}\right] }
\end{aligned}
$$

is called the observation vector. In the context of this paper, fixed feedback compensators $K$ will be considered. The input to the feedback compensator is given by the performance variable, therefore $y_{2}(t)=e^{0}(t)$. Its output will be $u_{2}(t)=$ $K \cdot y_{2}(t)$. The unmeasurable value of the output of the primary path (when the compensation is active) is denoted $x(t)$. The a priori output of the secondary path is denoted $\hat{z}^{0}(t+1)=\hat{z}(t+1 \mid \hat{\boldsymbol{\Theta}}(t))$ while its input is $\hat{u}(t)$. One has

$$
\hat{z}^{0}(t+1)=\frac{B_{G}^{*}\left(q^{-1}\right)}{A_{G}\left(q^{-1}\right)} \hat{u}(t)=\frac{B_{G}^{*}\left(q^{-1}\right)}{A_{G}\left(q^{-1}\right)} \hat{u}(t \mid \hat{\boldsymbol{\Theta}}(t)) .
$$

The measured residual acceleration (or force) satisfies the following equation

$$
e^{0}(t+1)=x(t+1)+\hat{z}^{0}(t+1) .
$$

The filtered a priori adaptation error is defined as

$$
\begin{aligned}
& \nu^{0}(t+1)=\nu(t+1 \mid \hat{\boldsymbol{\Theta}}(t))= \\
& =\varepsilon^{0}(t+1)+\sum_{i=1}^{n_{1}} v_{i}^{B} \varepsilon(t+1-i)-\sum_{i=1}^{n_{2}} v_{i}^{A} \nu^{0}(t+1-i),
\end{aligned}
$$

where

$$
\begin{aligned}
& \varepsilon^{0}(t+1)=\varepsilon(t+1 \mid \hat{\boldsymbol{\Theta}}(t))=-e^{0}(t+1)=-x(t+1)-\hat{z}^{0}(t+1), \\
& \varepsilon(t+1)=\varepsilon(t+1 \mid \hat{\mathbf{\Theta}}(t+1))=-e(t+1)=-x(t+1)-\hat{z}(t+1)
\end{aligned}
$$

\footnotetext{
${ }^{1}$ In adaptive control and estimation the predicted output at $t+1$ can be computed either on the basis of the previous parameter estimates (a priori, time $t$ ) or on the basis of the current parameter estimates (a posteriori, time $t+1)$.
}

are also called, respectively, the a priori and the a posteriori unfiltered adaptation errors.

The coefficients $v_{i}^{X}, X \in\{B, A\}$, are the coefficients of an IIR filter, with all poles and zeros inside the unit circle, acting on the adaptation error

$$
\begin{aligned}
V\left(q^{-1}\right) & =\frac{B_{V}\left(q^{-1}\right)}{A_{V}\left(q^{-1}\right)}, \\
X_{V}\left(q^{-1}\right) & =1+q^{-1} X_{V}^{*}\left(q^{-1}\right)=1+\sum_{i=1}^{n_{j}} v_{i}^{X} q^{-i},
\end{aligned}
$$

where $X \in\{B, A\}$. The filtered a posteriori unmeasurable (but computable) adaptation error is given by

$$
\begin{aligned}
& \nu(t+1)=\nu(t+1 \mid \hat{\boldsymbol{\Theta}}(t+1))= \\
& =\varepsilon(t+1)+\sum_{i=1}^{n_{1}} v_{i}^{B} \varepsilon(t+1-i)-\sum_{i=1}^{n_{2}} v_{i}^{A} \nu(t+1-i),
\end{aligned}
$$

with $\varepsilon(t+1)$ given in 21.

The a posteriori value of the output of the secondary path $\hat{z}(t+1)$ (dummy variable) is given by

$$
\hat{z}(t+1)=\hat{z}(t+1 \mid \hat{\mathbf{\Theta}}(t+1))=\frac{B_{G}^{*}\left(q^{-1}\right)}{A_{G}\left(q^{-1}\right)} \hat{u}(t \mid \hat{\boldsymbol{\Theta}}(t+1)) .
$$

For compensators with constant parameters: $\nu^{0}(t)=\nu(t)$, $\varepsilon^{0}(t)=\varepsilon(t), e^{0}(t)=e(t), \hat{z}^{0}(t)=\hat{z}(t), \hat{u}^{0}(t)=\hat{u}(t)$. Remark: when $V\left(q^{-1}\right)=1$ (the adaptation error is not filtered) the a priori and the a posteriori adaptation errors will have respectively the forms

$$
\begin{aligned}
& \nu^{0}(t+1)=\nu(t+1 \mid \hat{\boldsymbol{\Theta}}(t))= \\
& \quad=\varepsilon(t+1 \mid \hat{\boldsymbol{\Theta}}(t))=-e^{0}(t+1)=-x(t+1)-\hat{z}^{0}(t+1)
\end{aligned}
$$

$$
\begin{aligned}
& \nu(t+1)=\nu(t+1 \mid \hat{\boldsymbol{\Theta}}(t+1))=\varepsilon(t+1 \mid \hat{\boldsymbol{\Theta}}(t+1))= \\
& =-e(t+1)=-x(t+1)-\hat{z}(t+1) .
\end{aligned}
$$

\section{Development of the Algorithms}

The algorithms for adaptive feedforward compensation in the presence of a feedback controller will be developed under the following hypotheses:

H1) The signal $w(t)$ is bounded, i.e.,

$$
|w(t)| \leq \alpha, \quad \forall t \quad(0 \leq \alpha<\infty)
$$

(which is equivalent to say that $s(t)$ is bounded and $W\left(q^{-1}\right)$ in Figure 3 is asymptotically stable).

H2) Perfect matching condition - There exists a filter $N\left(q^{-1}\right)$ of finite dimension such that

$$
\frac{N\left(z^{-1}\right)}{1-N\left(z^{-1}\right) M\left(z^{-1}\right)} G\left(z^{-1}\right)=-D\left(z^{-1}\right)
$$

and the characteristic polynomials:

- of the "internal" positive coupling loop

$$
P\left(z^{-1}\right)=A_{M}\left(z^{-1}\right) S\left(z^{-1}\right)-B_{M}\left(z^{-1}\right) R\left(z^{-1}\right),
$$

- of the closed loop (G-K) 
$P_{c l}\left(z^{-1}\right)=A_{G}\left(z^{-1}\right) A_{K}\left(z^{-1}\right)+B_{G}\left(z^{-1}\right) B_{K}\left(z^{-1}\right)$,

- and of the coupled feedforward-feedback loop

$$
P_{f b-f f}=A_{M} S\left[A_{G} A_{K}+B_{G} B_{K}\right]-B_{M} R A_{K} A_{G}
$$

are Hurwitz.

H3) Deterministic context - The effect of the measurement noise upon the measured residual error is neglected.

H4) The primary path model $D\left(z^{-1}\right)$ is unknown and constant.

Once the algorithms are developed under these hypotheses, $\mathrm{H} 2$ and $\mathrm{H} 3$ can be removed and the algorithms can be analyzed in this modified context.

A first step in the development of the algorithms is to establish a relation between the errors on the estimation of the parameters of the feedforward filter (with respect to the optimal values) and the measured unfiltered adaptation error $\epsilon(t+1)$. This is summarized in the following lemma.

Lemma 1: Let the system be described by eqs. (1) (25). Under hypotheses $H 1, H 2, H 3$, and $H 4$, using a feedforward compensator $\hat{N}$ with constant parameters, one has:

$$
\varepsilon(t+1 \mid \hat{\boldsymbol{\Theta}})=\frac{A_{M} A_{G} A_{K} G}{P_{f b-f f}}[\boldsymbol{\Theta}-\hat{\boldsymbol{\Theta}}]^{T} \boldsymbol{\Phi}(t),
$$

where $\Theta$ (the vector of parameters of the optimal filter $N$ assuring perfect matching) is given by (8),

$$
\hat{\boldsymbol{\Theta}}^{T}=\left[\hat{s}_{1}, \ldots \hat{s}_{n_{S}}, \hat{r}_{0} \ldots \hat{r}_{n_{R}}\right]=\left[\hat{\mathbf{\Theta}}_{S}^{T}, \hat{\boldsymbol{\Theta}}_{R}^{T}\right]
$$

is the vector of constant estimated parameters of $\hat{N}$, and $\boldsymbol{\Phi}(t)$ has been defined in 16 .

The proof of this lemma has been given in [8]. Filtering the vector $\boldsymbol{\Phi}(t)$ with an asymptotically stable filter $L\left(q^{-1}\right)=$ $\frac{B_{L}}{A_{L}}$, eq. (33) for $\hat{\boldsymbol{\Theta}}=$ constant leads to

$$
\begin{gathered}
\varepsilon(t+1 \mid \hat{\boldsymbol{\Theta}})=\frac{A_{M} A_{G} A_{K} G}{P_{f b-f f} L}[\boldsymbol{\Theta}-\hat{\boldsymbol{\Theta}}]^{T} \boldsymbol{\Phi}_{f}(t), \\
\boldsymbol{\Phi}_{f}(t)=L\left(q^{-1}\right) \boldsymbol{\Phi}(t),
\end{gathered}
$$

and filtering $\varepsilon(t+1 \mid \hat{\boldsymbol{\Theta}})$ through the filter $V\left(q^{-1}\right)$ one gets

$$
\nu(t+1 \mid \hat{\boldsymbol{\Theta}})=\frac{A_{M} A_{G} A_{K} G V}{P_{f b-f f} L}[\boldsymbol{\Theta}-\hat{\boldsymbol{\Theta}}]^{T} \boldsymbol{\Phi}_{f}(t) .
$$

Eq. (37) will be used to develop the adaptation algorithms, neglecting for the moment the non-commutativity of the operators when $\hat{\Theta}$ is time varying (however an exact algorithm can be derived in such cases - see [14]). Replacing the fixed estimated parameters by the current estimated parameters,

\footnotetext{
${ }^{2}$ However, one should take into account that a change of notations has been done with respect to [8]. The following enumeration gives the correspondance in notation between the present article and [8]: $w(t) \leftrightarrow d(t)$, $\hat{u}_{1}(t) \leftrightarrow \hat{y}_{1}(t), \hat{u}(t) \leftrightarrow \hat{y}(t), \hat{y}_{1}(t) \leftrightarrow \hat{u}(t)$, and $e^{0}(t) \leftrightarrow \chi^{0}(t)$
}

eq. (35) becomes the equation of the a posteriori residual filtered adaptation error $\nu(t+1)$ (which is computed)

$$
\nu(t+1)=\frac{A_{M} A_{G} A_{K} V}{P_{f b-f f} L} G[\boldsymbol{\Theta}-\hat{\boldsymbol{\Theta}}(t+1)]^{T} \boldsymbol{\Phi}_{f}(t) .
$$

Eq. (38) has the standard form for an a posteriori adaptation error ([14]), which allows to introduce the general adaptation algorithm including filtering of the residual error and of the observations as well as the "Integral + Proportional" adaptation suggests to use the following IP-PAA:

$$
\begin{aligned}
& \hat{\boldsymbol{\Theta}}_{\mathbf{I}}(t+1)=\hat{\boldsymbol{\Theta}}_{\mathbf{I}}(t)+\xi(t) \mathbf{F}_{\mathbf{I}}(t) \mathbf{\Psi}(t) \nu(t+1), \\
& \hat{\boldsymbol{\Theta}}_{\mathbf{P}}(t+1)=\mathbf{F}_{\mathbf{P}}(t) \boldsymbol{\Psi}(t) \nu(t+1), \\
& \varepsilon(t+1)=\frac{\varepsilon^{0}(t+1)}{1+\mathbf{\Psi}^{T}(t)\left(\xi(t) \mathbf{F}_{\mathbf{I}}(t)+\mathbf{F}_{\mathbf{P}}(t)\right) \mathbf{\Psi}(t)}, \\
& \nu(t+1)=\varepsilon(t+1)+\sum_{i=1}^{n_{1}} v_{i}^{B} \varepsilon(t+1-i)- \\
& -\sum_{i=1}^{n_{2}} v_{i}^{A} \nu(t+1-i) \\
& \mathbf{F}_{\mathbf{I}}(t+1)=\frac{1}{\lambda_{1}(t)}\left[\mathbf{F}_{\mathbf{I}}(t)-\frac{\mathbf{F}_{\mathbf{I}}(t) \boldsymbol{\Psi}(t) \mathbf{\Psi}^{T}(t) \mathbf{F}_{\mathbf{I}}(t)}{\frac{\lambda_{1}(t)}{\lambda_{2}(t)}+\boldsymbol{\Psi}^{T}(t) \mathbf{F}_{\mathbf{I}}(t) \mathbf{\Psi}(t)}\right], \\
& \mathbf{F}_{\mathbf{P}}(t)=\alpha(t) \mathbf{F}_{\mathbf{I}}(t), \mathbf{F}_{\mathbf{I}}(0)=\gamma(0) \cdot \mathbf{I}, \alpha(t)>-0.5, \\
& \mathbf{F}(t)=\xi(t) \mathbf{F}_{\mathbf{I}}(t)+\mathbf{F}_{\mathbf{P}}(t) \\
& \xi(t)=1+\frac{\lambda_{2}(t)}{\lambda_{1}(t)} \boldsymbol{\Psi}^{T}(t) \mathbf{F}_{\mathbf{P}}(t) \boldsymbol{\Psi}(t), \\
& \hat{\boldsymbol{\Theta}}(t+1)=\hat{\boldsymbol{\Theta}}_{\mathbf{I}}(t+1)+\hat{\boldsymbol{\Theta}}_{\mathbf{P}}(t+1), \\
& 1 \geq \lambda_{1}(t)>0,0 \leq \lambda_{2}(t)<2, \mathbf{F}_{\mathbf{I}}(0)>0, \\
& \boldsymbol{\Psi}(t)=\boldsymbol{\Phi}_{f}(t),
\end{aligned}
$$

where $\nu(t+1)$ is the generalized filtered adaptation error (see also Section III for more details), $\lambda_{1}(t)$ and $\lambda_{2}(t)$ allow to obtain various profiles for the matrix adaptation gain $\mathbf{F}(t)$ ([14]), and $\gamma(0)$ is a positive scalar value. By taking $\lambda_{2}(t) \equiv 0$ one obtains a constant adaptation gain matrix and choosing $\mathbf{F}_{\mathbf{I}}=\gamma \mathbf{I}, \gamma>0$ one gets a scalar adaptation gain). For $\alpha(t) \equiv 0$, one obtains the algorithm with integral adaptation gain introduced in [4]. For the adaptive operation, an $\mathbf{F}_{\mathbf{I}}(t)$ with constant trace can be obtained by automatically computing $\lambda_{1}(t)$ and $\lambda_{2}(t)$ at each sampling period as a function of the newly computed trace of the "Integral" adaptation matrix, $\operatorname{tr}\left(\mathbf{F}_{\mathbf{I}}(t)\right)$, and the desired constant trace, $\operatorname{tr}\left(\mathbf{F}_{I_{0}}\right)$. In this case, a design parameter $\alpha_{F}=\frac{\lambda_{1}(t)}{\lambda_{2}(t)}$ (chosen equal to 1 in Section VIII is also used. The equations are given below:

$$
\lambda_{1}(t)=\frac{\operatorname{tr}\left(\mathbf{F}_{\mathbf{I}}(t)\right)}{\operatorname{tr}\left(\mathbf{F}_{I_{0}}\right)}, \quad \lambda_{2}(t)=\frac{\lambda_{1}(t)}{\alpha_{F}} .
$$

Note also that eq. $39 \mathrm{e}$ is obtained from

$$
\mathbf{F}_{\mathbf{I}}^{-1}(t+1)=\lambda_{1}(t) \mathbf{F}_{\mathbf{I}}^{-1}(t)+\lambda_{2}(t) \boldsymbol{\Psi}(t) \boldsymbol{\Psi}^{T}(t),
$$


using the matrix inversion lemma ([14]). In Table \, several versions of the algorithms particularized for various choices of $V$ and $L$ are given.

In the last line of Table \,

$$
\hat{P}_{f b-f f}=\hat{A}_{M} \hat{S}\left[\hat{A}_{G} A_{K}+\hat{B}_{G} B_{K}\right]-\hat{B}_{M} \hat{R} A_{K} \hat{A}_{G}
$$

is an estimation of the characteristic polynomial of the coupled feedforward-feedback loop computed on the basis of available estimates of the parameters of the filter $\hat{N}$.

For the Algorithms $I I I$, several options for updating $\hat{P}_{f b-f f}$ can be considered:

- Run one of the Algorithms $I$ or $I I$ for a certain time to get estimates of $\hat{R}$ and $\hat{S}$;

- Run a simulation (using the identified models);

- Update $\hat{P}_{f b-f f}$ at each sampling instant or from time to time using Algorithm III (after a short initialization horizon using one of the Algorithms $I$ or $I I$ ).

\section{Analysis of the Algorithms}

\section{A. Deterministic case} form

The equation for the a posteriori adaptation error has the

$$
\nu(t+1)=H\left(q^{-1}\right)[\boldsymbol{\Theta}-\hat{\boldsymbol{\Theta}}(t+1)]^{T} \boldsymbol{\Psi}(t)
$$

where

$$
H\left(q^{-1}\right)=\frac{A_{M} A_{G} A_{K}}{P_{f b-f f}} \frac{G V}{L}, \quad \boldsymbol{\Psi}=\boldsymbol{\Phi}_{f} .
$$

Neglecting the non-commutativity of the time varying operators, one has the following result

Theorem 1: Assuming that eq. (43) represents the evolution of the a posteriori adaptation error and that the IP-PAA (39) is used, one has:

$$
\begin{array}{r}
\lim _{t \rightarrow \infty} \nu(t+1)=0 \\
\lim _{t \rightarrow \infty} \frac{\left[\nu^{0}(t+1)\right]^{2}}{1+\boldsymbol{\Psi}(t)^{T} \mathbf{F}(t) \boldsymbol{\Psi}(t)}=0 \\
\|\mathbf{\Psi}(t)\| \text { is bounded } \\
\lim _{t \rightarrow \infty} \nu^{0}(t+1)=0
\end{array}
$$

for any bounded initial conditions $\hat{\boldsymbol{\Theta}}(0), \quad \nu^{0}(0), \quad \mathbf{F}(0)$, provided that

$$
H^{\prime}\left(z^{-1}\right)=H\left(z^{-1}\right)-\frac{\lambda_{2}}{2}, \max _{t} \lambda_{2}(t) \leq \lambda_{2}<2, \forall t
$$

is a SPR transfer function.

The proof follows [15], [4], [9] and is omitted.

\section{B. Relaxing the positive real condition by IP adaptation}

The adaptive system formed by eq. (43) and the adaptation algorithm 39 admits an equivalent feedback representation (EFR). It is shown in (see [9])that using IP adaptation the exceess of passivity which is introduced by proportional adaptation can be transferred to the linear path and relax the positive real condition on 49

\section{COMPARISON With OTHER Algorithms}

The algorithms introduced in this paper are compared with the algorithms presented in [4], [16], [11], the FULMS ([17]), and the SHARF ([10]). Table gives a synthetic comparison.

\section{EXPERIMENTAL RESULTS}

The experiments considered in this paper concern the system described in Section III The objectives of the experiments to be presented in this paper are to illustrate (i) the advantages of using IP adaptation for performance improvement and (ii) the rationale for using filtering on the regressor and/or the filtering on the residual error. These aspects have not been yet discussed previously in the context of adaptive feedforward compensation. The frequency characteristics for the various identified models of the primary, secondary and reverse path and details of the identification procedure can be found in [4].

The feedback RS controller $K$ reduces the disturbance effect on the residual acceleration $\left(e^{0}(t)\right)$ where the secondary path $G$ has enough gain.

The adaptive feedforward filter structure for all of the experiments has been $n_{R}=3, n_{S}=4$ (total of 8 parameters). This complexity does not allow to verify the "perfect matching condition" (not enough parameters). The feedback controller has been active. A PRBS excitation on the global primary path has been considered as the disturbance. Several experiments have been conducted:

- I adaptation with filtering of the regressor;

- IP adaptation with filtering of the regressor;

- IP adaptation with filtering of the regressor and of the adaptation error.

For the adaptive operation $3^{3}$ the Algorithms $I_{L}$ and $I_{L V}$ have been used 4 Time domain results obtained on the AVC system with only an "Integral" PAA are shown in Figure 4 Figure 5 shows the time domain result obtained using the IP-PAA. The advantage of using an IP-PAA is an overall improvement of the transient behavior. In Figure 6 , in addition to the IPPAA a filtering of the adaptation error using $V\left(q^{-1}\right)=1-$ $0.9 q^{-1}$ has been introduced (which introduces a weight in high frequencies), using Algorithm $I_{L V}$.

In terms of global attenuation the three algorithms provide respectively $14.89 \mathrm{~dB} 15.42$ and $22.12 \mathrm{~dB}$

It is clear that "Integral + Proportional" adaptation gives better results than only "Integral" adaptation and that using a appropriate filtering of the adaptation error the results can be further improved

\section{CONCLUSIONS}

The paper has considered a general structure for the adaptive feedforward compensation in the presence of an internal positive feedback and of a feedback controller. Experimental evaluation on an active vibration control system has shown

\footnotetext{
${ }^{3}$ Algorithms $I_{V}-I I I_{V}$ can not be used in our case since the secondary path has unstable zeros.

${ }^{4}$ Algorithms $I I_{L}$ and $I I_{L V}$ can also be used, but in this particular case, the filters used in algorithms $I_{L}$ and $I_{L V}$ allow to satisfy the positive real condition.
} 


\begin{tabular}{|c|c|c|c|c|c|}
\hline \multicolumn{2}{|c|}{ Regressor filtering } & \multicolumn{2}{|c|}{ Error filtering } & \multicolumn{2}{c|}{ Double filtering } \\
\hline Alg. & Filter $L(V=1)$ & Alg. & Filter $V(L=1)$ & Alg. & Filters $(V$ and $L)$ \\
\hline \hline$I_{L}$ & $L=\hat{G}$ & $I_{V}$ & $V=\frac{1}{\hat{G}}$ & $I_{L V}$ & $L=\hat{G}, V \neq 1$ \\
\hline$I I_{L}$ & $L=\frac{G}{1+\hat{G} K}$ & $I I_{V}$ & $V=\frac{1+G K}{\hat{G}}$ & $I I_{L V}$ & $L=\frac{G}{1+\hat{G} K}, V \neq 1$ \\
\hline$I I I_{L}$ & $L=\frac{\hat{A}_{M} \hat{A}_{G} A_{K}}{\hat{P}_{f b-f f}} \hat{G}$ & $I I I_{V}$ & $V=\frac{\hat{P}_{f b-f f}}{\hat{A}_{M} \hat{A}_{G} A_{K} \hat{G}}$ & $I I I_{L V}$ & $L=\frac{\hat{A}_{M} \hat{A}_{G} A_{K}}{\hat{P}_{f b-f f}} \hat{G}, V \neq 1$ \\
\hline
\end{tabular}

TABLE I

ADAPTATION ALGORITHMS WITH REGRESSOR VECTOR AND/OR RESIDUAL ERROR FILTERING.

\begin{tabular}{|c|c|c|c|c|c|c|c|c|}
\hline \multirow{2}{*}{ Reference } & \multicolumn{2}{|c|}{ Adaptation } & \multicolumn{2}{|c|}{ Adaptation gain } & \multicolumn{2}{|c|}{ Filtering } & \multicolumn{2}{|c|}{ Taking into account } \\
\hline & IP & I & Matrix & Scalar & Regressor vector & Residual error & Local feedback & Internal feedback \\
\hline Present paper & yes & yes & $\overline{\text { yes }}$ & yes & yes & yes & yes & $\overline{\text { yes }}$ \\
\hline [4] & no & yes & yes & yes & yes & no & no & yes \\
\hline [8] & no & yes & yes & yes & yes & no & yes & yes \\
\hline [16] & no & yes & no & yes & no & no & no & yes \\
\hline FULMS & no & yes & no & yes & yes & no & no & yes \\
\hline SHARF [10] & no & yes & no & yes & no & yes & no & no \\
\hline [11] & no & yes & yes & no & yes & yes & no & no \\
\hline
\end{tabular}

TABLE II

COMPARISON OF ALGORITHMS FOR ADAPTIVE FEEDFORWARD COMPENSATION IN AVC WITH MECHANICAL COUPLING.

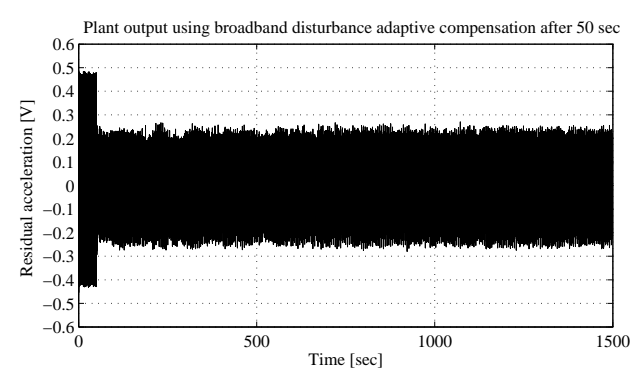

Fig. 4. Real time results obtained with Algorithm $I_{L}$ using "Integral" scalar adaptation gain.

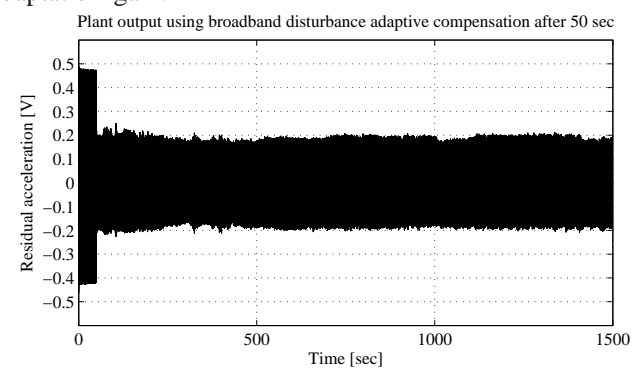

Fig. 5. Real time results obtained with Algorithm $I_{L}$ using "Integral + Proportional" scalar adaptation gain.

that adding appropriate filtering of the residual error and proportional adaptation can improve the global performance of the system.

\section{REFERENCES}

[1] S. Elliott, T. Sutton, Performance of feedforward and feedback systems for active control, Speech and Audio Processing, IEEE Transactions on 4 (3) (1996) $214-223$.

[2] S. Kuo, D. Morgan, Active noise control: a tutorial review, Proceedings of the IEEE 87 (6) (1999) 943 - 973.

[3] J. Zeng, R. de Callafon, Recursive filter estimation for feedforward noise cancellation with acoustic coupling, Journal of Sound and Vibration 291 (3-5) (2006) 1061 - 1079.

[4] I. Landau, M. Alma, T. Airimiţoaie, Adaptive feedforward compensation algorithms for active vibration control with mechanical coupling, Automatica 47 (10) (2011) 2185 - 2196.

[5] R. A. de Callafon, C. E. Kinney, Robust estimation and adaptive controller tuning for variance minimization in servo systems, Journal of Advanced Mechanical Design, Systems, and Manufacturing 4 (1) (2010) 130-142.

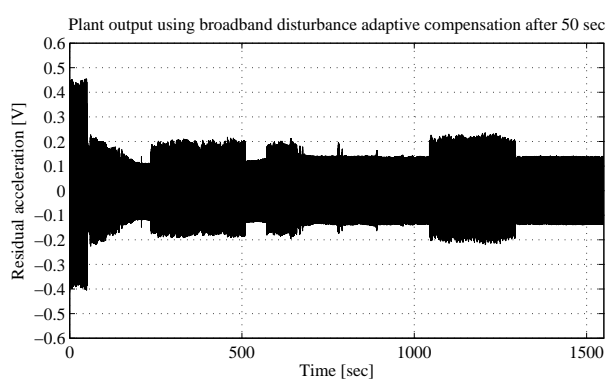

Fig. 6. Real time results obtained with Algorithm $I_{L V}$ using "Integral + Proportional" scalar adaptation gain and adaptation error filtering.

[6] L. Ray, J. Solbeck, A. Streeter, R. Collier, Hybrid feedforwardfeedback active noise reduction for hearing protection and communication, The Journal of the Acoustical Society of America 120 (4) (2006) 2026-2036.

[7] E. Esmailzadeh, A. Alasty, A. Ohadi, Hybrid active noise control of a one-dimensional acoustic duct, Journal of vibration and acoustics 124 (1) (2002) 10-18.

[8] M. Alma, I. D. Landau, T.-B. Airimitoaie, Adaptive feedforward compensation algorithms for avc systems in the presence of a feedback controller, Automatica 48 (5) (2012) 982 - 985.

[9] T.-B. Airimiţoaie, I. D. Landau, Improving adaptive feedforward vibration compensation by using "integral+proportional" adaptation, Automatica 49 (5) (2013) 1501-1505.

[10] M. Larimore, J. Treichler, J. Johnson, C., Sharf: An algorithm for adapting iir digital filters, Acoustics, Speech and Signal Processing, IEEE Transactions on 28 (4) (1980) 428 - 440.

[11] A. Montazeri, J. Poshtan, A new adaptive recursive rls-based fastarray iir filter for active noise and vibration control systems, Signal Processing 91 (1) (2011) 98 - 113.

[12] X. Sun, D.-S. Chen, A new infinte impulse response filter-based adaptive algorithm for active noise control, Journal of Sound and Vibration 258 (2) (2002) 385 - 397.

[13] X. Sun, G. Meng, Steiglitzmcbride type adaptive iir algorithm for active noise control, Journal of Sound and Vibration 273 (1-2) (2004) $441-450$.

[14] I. D. Landau, R. Lozano, M. M'Saad, A. Karimi, Adaptive control, 2nd Edition, Springer, London, 2011.

[15] I. Landau, An extension of a stability theorem applicable to adaptive control, Automatic Control, IEEE Transactions on 25 (4) (1980) 814 $-817$.

[16] C. Jacobson, J. Johnson, C.R., D. McCormick, W. Sethares, Stability of active noise control algorithms, Signal Processing Letters, IEEE 8 (3) (2001) $74-76$.

[17] A. Wang, W. Ren, Convergence analysis of the filtered-u algorithm for active noise control, Signal Processing 83 (2003) 1239-1254. 